\title{
Desenvolvimento e Estudo de Estabilidade Físico-Química de Formulações Cosméticas Antienvelhecimento
}

\author{
Tainara Fátima da Silva ${ }^{1}$, Josiane Woutheres Bortolotto ${ }^{2}$, Regis Augusto Norbert Deuschle ${ }^{3}$ \\ Thiago de Souza Claudino ${ }^{4}$, Viviane Cecilia Kessler Nunes Deuschle ${ }^{5}$
}

\begin{abstract}
RESUMO
A pele é o maior órgão do corpo humano e encontra-se em contato direto com fatores ambientais, devendo, desta forma, ser continuamente protegida. Este trabalho teve como objetivo o desenvolvimento de emulsões antienvelhecimento contendo óleo de romã e vitamina $\mathrm{E}$ e determinação da estabilidade destas formulações. Foram preparadas três formulações: formulação 1: contendo $3 \%$ de óleo de romã; formulação 2: contendo $2 \%$ de vitamina $E$; formulação 3 : contendo $3 \%$ óleo de romã e $2 \%$ vitamina E. Durante 90 dias as amostras foram submetidas a diferentes condições de temperatura e foram analisados os seguintes parâmetros: características organolépticas, $\mathrm{pH}$ e viscosidade. Observou-se que a amostra que continha apenas vitamina E permaneceu estável em todos os ensaios realizados no período de 90 dias. Em todas as amostras mantidas em temperatura ambiente não houve reduções na viscosidade. As demais formulações apresentaram pequenas alterações em relação às características organolépticas. Conclui-se que a formulação contendo apenas vitamina E demonstrou maior estabilidade em relação às demais.
\end{abstract}

Palavras-chave: Óleo de romã. Vitamina E. Cosmético. Pele. Antioxidante.

\section{DEVELOPMENT AND STABILITY STUDIES OF ANTI-AGING COSMETIC FORMULATIONS}

\begin{abstract}
The skin is the largest organ of the human body and is in direct contact with environmental factors, and, in this way, must be continuously protected. This work aimed to the development of antiaging emulsions containing pomegranate oil and vitamin $\mathrm{E}$ and to determine the stability of these formulations. Three formulations were prepared: formulation 1: containing $3 \%$ pomegranate oil; formulation 2: $2 \%$ vitamin E; Formulation 3: $3 \%$ pomegranate oil and $2 \%$ vitamin E. For ninety days the samples were submitted to different temperature conditions (low, ambient and high) and the following parameters were analyzed: organoleptic characteristics, $\mathrm{pH}$ and viscosity. It was observed that the sample containing only vitamin $\mathrm{E}$ remained stable for all tests carried out during the ninety day period. In all samples kept at room temperature there were no reductions in viscosity. The other formulations presented small changes in relation to the organoleptic characteristics. It was concluded that the formulation containing only vitamin $\mathrm{E}$ showed greater stability in relation to the others.
\end{abstract}

Keywords: Pomegranate oil. Vitamin E. Cosmetic. Skin. Antioxidant.

Recebido em: 18/1/2018

Alterações requeridas em: 4/3/2019

Aceito em: 9/3/2019

\footnotetext{
Graduada em Farmácia - Universidade de Cruz Alta (Unicruz). tainaraks@live.com

${ }^{2}$ Graduação em Farmácia com ênfase em Farmácia Industrial (Pontifícia Universidade Católica do Rio Grande do Sul). Mestrado em Ciências Biológicas: Bioquímica (UFRGS). Doutorado em Biologia Celular e Molecular (PUCRS - PPGBCM). Professora-adjunta da Universidade de Cruz Alta (Unicruz). bortolotto@unicruz.edu.br

${ }^{3}$ Graduação em Farmácia Bioquímica - Análises Clínicas e Mestrado em Ciência e Tecnologias Farmacêuticas pela Universidade Federal de Santa Maria UFSM. Docente da Unicruz. rdeuschle@unicruz.edu.br

${ }^{4}$ Graduação em Farmácia com ênfase em Farmácia Industrial, Mestrado em Ciências Farmacêuticas e Doutorado em Química pela Universidade Federal do Rio Grande do Sul. tclaudino@unicruz.edu.br

${ }^{5}$ Graduação em Farmácia Bioquímica pela Universidade de Cruz Alta - Unicruz. Mestrado e Doutorado em Ciências Farmacêuticas pela Universidade Federal de Santa Maria - UFSM. Professora-adjunta da Universidade de Cruz Alta.vdeuchle@unicruz.edu.br
} 


\section{INTRODUÇÃO}

A pele é o maior órgão do organismo humano e consiste em sua primeira linha de defesa. É dividida em duas camadas (epiderme e derme), seguidas da hipoderme ou tela subcutânea e é responsável por desempenhar importantes funções, como absorção, transpiração, perspiração, termorregulação, defesa, queratogênese, pigmentação, nutrição e proteção (BATISTELLA; CHORILLI; LEONARDI, 2007; ORESAJO et al., 2012; MOUAD; PORTO, 2014; SINIGAGLIA et al., 2014).

O processo de envelhecimento da pele ocorre de duas formas: envelhecimento intrínseco e extrínseco. O primeiro é inevitável e trata-se do envelhecimento natural e cronológico que o organismo sofre ao longo dos anos. Na pele é evidenciado pela diminuição na síntese de colágeno, elastina e outras macromoléculas importantes, sintetizadas pelos fibroblastos, células presentes na derme. Já o envelhecimento extrínseco é causado por fatores externos. O fator mais comumente associado ao envelhecimento extrínseco é a radiação solar, o que provoca o fotoenvelhecimento, uma vez que estimula a formação de espécies reativas e radicais livres, que podem acelerar o envelhecimento cutâneo (MOTTA; FIGUEIREDO; DUARTE, 2004; BATISTELLA; CHORILLI; LEONARDI, 2007; STRUTZEL et al., 2007; BARBA; RIBEIRO, 2009; KAMMEYER; LUTIEN, 2015).

O envelhecimento da pele e as formas de prevenção são assuntos amplamente abordados. É comum que a indústria cosmética venha desenvolvendo cada vez mais pesquisas para descobrir novas matérias-primas e seu uso principalmente em relação aos produtos de origem vegetal e derivados, com apelo de produtos sustentáveis (ISAAC et al., 2008; LYRIO et al., 2011; FARIA et al., 2012). Dessa forma, uma das estratégias para prevenir o envelhecimento precoce da pele é o desenvolvimento de formulações contendo substâncias antioxidantes, que apresentam capacidade de proteger as células e suas estruturas contra os danos provocados pela radiação solar ao longo dos anos (SOARES, 2002). Essas substâncias apresentam a capacidade de neutralizar as espécies reativas e radicais livres, doando-lhes um elétron a fim de estabilizá-los (SCHNEIDER; OLIVEIRA, 2004). Alguns exemplos são as formulações que usam matérias-primas vegetais, originárias de plantas, como os derivados da romã e também as vitaminas, como a $C$ e a $E$, que auxiliam na neutralização de radicais livres (DA ROSA, 2008).
O tocoferol, ou vitamina $E$, é uma vitamina lipossolúvel com ação antioxidante (BARREIROS; DAVID; DAVID, 2006; STRUTZEL et al., 2007). Na forma tópica, tem o poder de reduzir a ação de metalproteinases e impedir a ação dos radicais livres, protegendo a membrana e prevenindo a formação de rugas (TESTON; NARDINO; PIVATO, 2010; ALMEIDA et al., 2010; ALLEMANN; BAUMANN, 2012). Além disso, também é conhecido como um dos mais importantes inibidores da peroxidação lipídica nas células (GUARATINI; MEDEIROS; COLEPICOLO, 2007).

Já o óleo de romã, extraído das sementes da Punica granatum (romã), apresenta sua constituição rica em ácidos graxos poli-insaturados como o ácido linolênico e linoleico e outras gorduras, como os ácidos punícico, oleico, esteárico e palítico (OZGUL-YUCEL, 2005; FADAVI; BARZEGAR; AZIZI, 2005). Apresenta ação antioxidante (SILVA JUNIOR et al, 2013) e a presença do ácido punícico promove ação anti-inflamatória e antitumoral (VIUDA-MARTOS; FERNÁNDEZ-LÓPEZ; PÉREZ-ÁLVAREZ, 2010). O óleo de romã a uma concentração de $5 \%$ demonstrou reduzir significativamente a incidência de tumores em câncer de pele induzido em ratas, revelando um alto potencial como substância quimiopreventiva neste tipo de neoplasia (HORA et al., 2003).

Para que qualquer substância ativa apresente eficácia, no entanto, é necessário a realização da etapa de pesquisa e desenvolvimento de formulações, combinando matérias-primas compatíveis quimicamente e que permitam a sua permeação através das camadas da pele. Um dos passos determinantes no desenvolvimento de um cosmético é a determinação de sua estabilidade, com objetivo de prever alterações físicas, químicas ou microbiológicas (CHORILLI et al., 2007a; VIEIRA et al., 2009). Com isso, é possível certificar-se de que o produto final apresente um bom aspecto, odor, espalhabilidade, sensorial agradável à pele, $\mathrm{pH}$ e viscosidade adequados e que acima de tudo se mantenha estável durante o prazo de validade (CHORILLI et al., 2007b; BABY et al., 2008; VIEIRA et al., 2009).

A Agência Nacional de Vigilância Sanitária (Anvisa) publicou o Guia de Estabilidade de Produtos Cosméticos, que traz informações desde fatores que influenciam na estabilidade, acondicionamento de amostras, tipos de estabilidade e critérios para aprovação dos produtos (BRASIL, 2004). 
Nesse contexto, é objetivo deste trabalho o desenvolvimento de três formulações cosméticas contendo óleo de romã e vitamina $\mathrm{E}$, e estudar a sua estabilidade físico-química em diferentes condições de temperatura.

\section{MÉTODO}

As amostras foram preparadas no Laboratório de Farmacotécnica da Universidade de Cruz Alta, no ano de 2017. Foi preparada uma emulsão não iônica base do tipo óleo-água (O/A), de acordo com o Formulário Nacional, com adaptações (BRASIL, 2004), como descrito na Tabela 1.

Tabela 1 - Composição da emulsão base

\begin{tabular}{lc}
\hline \multicolumn{1}{c}{ Componente } & Quantidade (\%) \\
Fase oleosa & $13 \%$ \\
Polawax & $3 \%$ \\
Estearato de octila & $0,1 \%$ \\
BHT & \\
Fase aquosa & $0,2 \%$ \\
EDTA & $5,14 \%$ \\
Sorbitol & $75,8 \%$ \\
Água & \\
Conservantes & $0,1 \%$ \\
Solução de Parabenos & $0,61 \%$ \\
Germal & $2 \%$ \\
Silicone voltátil &
\end{tabular}

Fonte: Os autores.

Após, foram preparadas três diferentes formulações, às quais incorporou-se as substâncias ativas e designadas como segue: Formulação 1: contendo 3\% de óleo de romã; Formulação 2: contendo $2 \%$ de vitamina $\mathrm{E}$; Formulação 3: contendo $3 \%$ de óleo de romã e $2 \%$ de vitamina $E$.

A determinação da estabilidade físico-química foi efetuada conforme especificações do Guia de Controle de Qualidade de Produtos Cosméticos (BRASIL, 2008) e Guia de Estabilidade de Produtos Cosméticos (BRASIL, 2004).

Antes do início dos estudos de estabilidade foi realizado o teste de centrifugação com amostras das formulações desenvolvidas. As formulações foram colocadas em tubos Falcon de $15 \mathrm{~mL}$ e submetidas a um ciclo de $3.000 \mathrm{rpm}$ durante 30 minutos. A seguir as amostras foram analisadas visualmente levando em consideração seu aspecto, cor, odor, brilho e separação de fases ou qualquer sinal de possível instabilidade (BRASIL, 2004).

Para a realização do ensaio de estabilidade acelerada, as amostras das formulações foram fracionadas em três frascos plásticos, bem vedados e armazenados em baixa temperatura $\left(5 \pm 2^{\circ} \mathrm{C}\right)$, temperatura ambiente $(25 \pm 2 \circ \mathrm{C})$ e em alta temperatura $(45 \pm 2 \circ \mathrm{O})$, durante um período de 90 dias, utilizando os seguintes parâmetros como critérios de avaliação: verificação das características sensoriais (aspecto, cor e odor), de acordo com escala representada na Tabela 2, determinação da viscosidade e do $\mathrm{pH}$. As análises foram realizadas nos tempos 0, 7, 30, 60 e 90 dias (BRASIL, 2004). Todas as amostras foram ambientadas à temperatura ambiente antes da realização dos ensaios e, após as análises, todas retornaram para suas respectivas condições de armazenamento até o fim do experimento.

Tabela 2 - Escala representativa do aspecto das formulações cosméticas

\begin{tabular}{|c|c|}
\hline ESCALA & ASPECTO \\
\hline 1 & $\begin{array}{l}\text { Nenhuma alteração visível - sem alteração } \\
\text { de cor ou aspecto }\end{array}$ \\
\hline 2 & Leve alteração de coloração/odor \\
\hline 3 & Perda total de coloração/odor \\
\hline 4 & $\begin{array}{l}\text { Evidência de falta de homogeneidade e alte- } \\
\text { ração no aspecto }\end{array}$ \\
\hline
\end{tabular}

A viscosidade das formulações foi avaliada com o auxílio de um viscosímetro rotacional Brookfield, com spindle $\mathrm{S64}$. Os valores foram obtidos após $1 \mathrm{mi}-$ nuto de rotação do spindle, na velocidade de 0,3 rpm e expressos em porcentagem (\%) de redução de viscosidade em relação ao tempo zero. $\mathrm{O}$ pH foi determinado diretamente na formulação utilizando pHmetro, previamente calibrado com soluções tampão $\mathrm{pH} 4,00$ e pH 7,00 (BRASIL, 2004).

\section{RESULTADOS}

Após a adição dos ativos nas formulações, estas foram submetidas ao teste de centrifugação. Não houve alterações visíveis nas formulações, mostrando-se, desta forma, possível a continuação dos ensaios de estabilidade.

Os resultados obtidos por meio da verificação das características sensoriais para as três formulações encontram-se demonstrados na Tabela 3 e os parâmetros utilizados encontram-se na Tabela 2. 
Tabela 3 - Características sensoriais das formulações nas diferentes condições e durante período de armazenagem

\begin{tabular}{|c|c|c|c|c|c|c|c|c|c|}
\hline \multirow[t]{2}{*}{$\begin{array}{l}\text { Tempo/ } \\
\text { Dias }\end{array}$} & \multicolumn{3}{|c|}{$\begin{array}{l}\text { Formulação } 1 \\
\text { (Óleo de Romã) }\end{array}$} & \multicolumn{3}{|c|}{$\begin{array}{c}\text { Formulação } 2 \\
\text { (Vitamina E) }\end{array}$} & \multicolumn{3}{|c|}{$\begin{array}{c}\text { Formulação } 3 \\
\text { (Vitamina E + Óleo de Romã) }\end{array}$} \\
\hline & $\left(5 \pm 2^{\circ} \mathrm{C}\right)$ & $\left(25 \pm 2^{\circ} \mathrm{C}\right)$ & $\left(45 \pm 2^{\circ} \mathrm{C}\right)$ & $\left(5 \pm 2^{\circ} \mathrm{C}\right)$ & $\left(25 \pm 2^{\circ} \mathrm{C}\right)$ & $\left(45 \pm 2^{\circ} \mathrm{C}\right)$ & $\left(5 \pm 2^{\circ} \mathrm{C}\right)$ & $\left(25 \pm 2^{\circ} \mathrm{C}\right)$ & $\left(45 \pm 2^{\circ} \mathrm{C}\right)$ \\
\hline Zero & 1 & 1 & 1 & 1 & 1 & 1 & 1 & 1 & 1 \\
\hline 7 & 1 & 1 & 1 & 1 & 1 & 1 & 1 & 1 & 1 \\
\hline 30 & 1 & 1 & 2 & 1 & 1 & 1 & 1 & 1 & 2 \\
\hline 60 & 1 & 1 & 2 & 1 & 1 & 1 & 1 & 1 & 2 \\
\hline 90 & 1 & 1 & 2 & 1 & 1 & 1 & 1 & 1 & 2 \\
\hline
\end{tabular}

Fonte: Dados da pesquisa.

Tabela 4 - Determinação do pH das formulações nas diferentes condições e durante período de armazenagem

\begin{tabular}{|c|c|c|c|c|c|c|c|c|c|}
\hline \multirow[t]{2}{*}{$\begin{array}{l}\text { Tempo/ } \\
\text { Dias }\end{array}$} & \multicolumn{3}{|c|}{$\begin{array}{l}\text { Formulação } 1 \\
\text { (Óleo de Romã) }\end{array}$} & \multicolumn{3}{|c|}{$\begin{array}{l}\text { Formulação } 2 \\
\text { (Vitamina E) }\end{array}$} & \multicolumn{3}{|c|}{$\begin{array}{c}\text { Formulação } 3 \\
\text { (Vitamina E + Óleo de Romã) }\end{array}$} \\
\hline & $\left(5 \pm 2^{\circ} \mathrm{C}\right)$ & $\left(25 \pm 2^{\circ} \mathrm{C}\right)$ & $\left(45 \pm 2^{\circ} \mathrm{C}\right)$ & $\left(5 \pm 2^{\circ} \mathrm{C}\right)$ & $\left(25 \pm 2^{\circ} \mathrm{C}\right)$ & $\left(45 \pm 2^{\circ} \mathrm{C}\right)$ & $\left(5 \pm 2^{\circ} \mathrm{C}\right)$ & $\left(25 \pm 2^{\circ} \mathrm{C}\right)$ & $\left(45 \pm 2^{\circ} \mathrm{C}\right)$ \\
\hline Zero & 4,6 & 4,6 & 4,6 & 4,6 & 4,6 & 4,6 & 4,6 & 4,6 & 4,6 \\
\hline 7 & 4,7 & 4,7 & 4,8 & 4,8 & 4,8 & 4,7 & 4,7 & 4,8 & 4,7 \\
\hline 30 & 4,7 & 4,6 & 4,6 & 4,6 & 4,6 & 4,6 & 4,6 & 4,7 & 4,6 \\
\hline 60 & 4,8 & 4,7 & 4,7 & 4,7 & 4,7 & 4,6 & 4,7 & 4,8 & 4,7 \\
\hline 90 & 4,9 & 4,8 & 4,8 & 4,7 & 4,8 & 4,7 & 4,7 & 4,8 & 4,7 \\
\hline
\end{tabular}

Fonte: Dados da pesquisa.

Tabela 5 - Determinação da viscosidade das formulações nas diferentes condições e durante período de armazenagem

\begin{tabular}{|c|c|c|c|c|c|c|c|c|c|}
\hline \multirow[t]{2}{*}{$\begin{array}{l}\text { Tempo/ } \\
\text { Dias }\end{array}$} & \multicolumn{3}{|c|}{$\begin{array}{c}\text { Formulação } 1 \\
\text { (Óleo de Romã) }\end{array}$} & \multicolumn{3}{|c|}{$\begin{array}{c}\text { Formulação } 2 \\
\text { (Vitamina E) }\end{array}$} & \multicolumn{3}{|c|}{$\begin{array}{c}\text { Formulação } 3 \\
\text { (Vitamina E + Óleo de Romã) }\end{array}$} \\
\hline & $\left(5 \pm 2^{\circ} \mathrm{C}\right)$ & $\left(25 \pm 2^{\circ} \mathrm{C}\right)$ & $\left(45 \pm 2^{\circ} \mathrm{C}\right)$ & $\left(5 \pm 2^{\circ} \mathrm{C}\right)$ & $\left(25 \pm 2^{\circ} \mathrm{C}\right)$ & $\left(45 \pm 2^{\circ} \mathrm{C}\right)$ & $\left(5 \pm 2^{\circ} \mathrm{C}\right)$ & $\left(25 \pm 2^{\circ} \mathrm{C}\right)$ & $\left(45 \pm 2^{\circ} \mathrm{C}\right)$ \\
\hline Zero & 0 & 0 & 0 & 0 & 0 & 0 & 0 & 0 & 0 \\
\hline 7 & 0 & 0 & 0 & 0 & 0 & 4,03 & 0 & 0 & 0 \\
\hline 30 & 0 & 0 & 54,73 & 0 & 0 & 0 & 0 & 0 & 0 \\
\hline 60 & 13,62 & 0 & 52,84 & 0 & 0 & 0 & 0 & 0 & 0,64 \\
\hline 90 & 0 & 0 & 0 & 3,22 & 0 & 0 & 29,87 & 0 & 33,11 \\
\hline
\end{tabular}

Os valores foram expressos em (\%) de redução de viscosidade em relação ao tempo zero para cada formulação.

Fonte: Dados da pesquisa.

A Tabela 4 representa os resultados obtidos na análise de $\mathrm{pH}$ durante o período de armazenamento das amostras.

Os resultados da verificação da viscosidade das formulações durante o período de armazenamento estão demonstrados na Tabela 5.

\section{DISCUSSÃO}

A pele sofre ao longo dos anos com muitos fatores externos aos quais fica exposta, sendo o principal deles a radiação solar, o que provoca a formação de espécies oxidativas e radicais livres e, consequentemente, o surgimento de rugas e manchas cutâneas (BARBA; RIBEIRO, 2009; KAMMEYER; LUTIEN, 2015).
Neste contexto, uma das abordagens de tratamento preventivo contra a formação dessas espécies é a utilização de produtos cosméticos contendo substâncias ativas antioxidantes, como a vitamina $E$ e o óleo de romã, retardando, dessa forma, o surgimento de rugas (SANTOS; OLIVEIRA, 2013; SILVA JUNIOR et al., 2013).

A vitamina $\mathrm{E}$ tem sido considerada um dos mais potentes antioxidantes, uma vez que apresenta a capacidade de manter a integridade de membranas e outros componentes biológicos, além de ser muito importante em tecidos com altos teores de ácidos graxos poli-insaturados, responsáveis por constituir membranas celulares altamente suscetíveis ao ataque de radicais livres. Entende-se, então, que a vitamina 
E age como um bloqueador das reações do processo de oxidação lipídica (STRUTZEL et al., 2007; TESTON; NARDINO; PIVATO, 2010; SANTOS, OLIVEIRA, 2013).

O óleo de romã apresenta potencial de utilização em formulações cosméticas como hidratante e antioxidante (SILVA JÚNIOR et al., 2013), dois aspectos essenciais na prevenção do envelhecimento da pele e também como anti-inflamatório e antitumoral (HORA et al., 2003).

Segundo o Guia de Estabilidade de Produtos Cosméticos da Anvisa, o formulador deve estabelecer os critérios e parâmetros utilizados nos estudos de estabilidade das formulações desenvolvidas, de acordo com as necessidades específicas do produto (BRASIL, 2004).

No primeiro teste realizado neste estudo, que se constitui da centrifugação das amostras, não houve separação de fases, o que é um sinal positivo para prosseguir com a formulação e os demais ensaios, indicando que a formulação é estável e homogênea. A separação de fases se dá por instabilidade na fórmula, que pode se originar de uma incompatibilidade entre matérias-primas e até mesmo devido a uma quantidade insuficiente de emulsionante ou pela evaporação da água da formulação (LANGE; HEBERLÉ; MILÃO, 2009).

Quanto à verificação das características sensoriais, a formulação com vitamina E permaneceu estável durante os 90 dias nas três temperaturas de armazenamento. Já as formulações que continham óleo de romã apresentaram uma leve alteração de cor e odor a partir do trigésimo dia, adquirindo um tom mais amarelado e um odor de rancificação, característico de processos oxidativos. Estas mudanças, entretanto, só foram percebidas nas amostras expostas a alta temperatura, o que, de certa forma, já era esperado, pois em temperatura elevada pode haver alterações em relação ao aspecto do produto, o que pode evidenciar decomposição de algum dos componentes da formulação (BRASIL, 2004).

Alterações nas características sensoriais do produto são de grande importância, pois ajudam a mensurar as mudanças que podem ocorrer até que o produto chegue ao consumidor, podendo definir se ele terá ou não uma boa aceitação. Mudanças nessas características decorrem de processos de desestabilização da formulação (ISAAC et al., 2008; FIGUEIREDO; MARTINI; MICHELIN, 2014).

As alterações de $\mathrm{pH}$ em formulações farmacêuticas e cosméticas são preocupantes, pois podem alterar toda a formulação desde o aspecto (mais ou me- nos viscoso) até a eficácia do princípio ativo utilizado, podendo, desta forma, não fornecer o resultado esperado, uma vez que alguns ativos não apresentam estabilidade em determinados $\mathrm{pH}$ e podem irritar a pele se não estiverem compatíveis com esta. Variações de $\mathrm{pH}$ em estudos de estabilidade podem ocorrer devido à degradação de componentes presentes na formulação (BUGNOTTO et al., 2006). Em relação ao pH das formulações, observou-se que os valores oscilaram entre 4,5 e 4,9 em todas as formulações, em todas as condições de temperaturas, evidenciando a preservação do produto. Além disso, essas variações foram mínimas e não comprometem o pH cutâneo, que fica entre 4,6 e 5,8 (LEONARDI; GASPAR; CAMPOS, 2002; HARRIS, 2012).

A viscosidade é a propriedade reológica mais estudada. Estudos reológicos são de suma importância para a área farmacêutica e cosmética, pois ajudam a avaliar inúmeras características de um medicamento de uso tópico ou formulação cosmética. É a reologia que engloba a verificação da viscosidade que, por sua vez, é uma expressão de resistência do fluido ao fluxo, ou seja, quanto maior a viscosidade, maior a resistência. Com isso, a viscosidade pode determinar a espalhabilidade da formulação na superfície da pele, a aceitabilidade do produto pelo consumidor e a facilidade ou não da formulação em sair da embalagem (CHORILLI et al., 2007b; ISAAC et al., 2008).

Quanto aos resultados referentes à viscosidade, a formulação contendo óleo de romã (Formulação 1), mantida em baixa temperatura apresentou uma redução em 60 dias (13,62\%), que se manteve após 90 dias de armazenamento. A amostra mantida em alta temperatura apresentou uma redução mais pronunciada, ou seja, em 30 e 60 dias de armazenamento. Sabe-se que a consistência das formulações apresenta tendência em sofrer reduções durante o armazenamento, indicando instabilidade (GUARATINI; GIANETI; CAMPOS, 2006).

A formulação contendo vitamina $E$ (Formulação 2) apresentou uma pequena queda de viscosidade após 60 dias, na amostra mantida em baixa temperatura. Já a formulação mantida em alta temperatura revelou uma redução em sua viscosidade em 7 dias de armazenamento, apresentando a maior queda em relação ao tempo zero. Essa condição é esperada quando as amostras são submetidas ao armazenamento em temperaturas mais elevadas.

Para a formulação que combina óleo de romã e vitamina $\mathrm{E}$ (Formulação 3), observou-se uma diminuição da viscosidade da formulação mantida em baixa temperatura em 90 dias de ensaio e na amostra em 
alta temperatura nos tempos de 60 e 90 dias. Verificou-se que todas as amostras mantidas em temperatura ambiente não sofreram redução de viscosidade ao longo do período de estudo, sendo a formulação que melhor manteve suas caraterísticas iniciais em relação a este parâmetro. Da mesma forma, notou-se que as formulações armazenadas em alta temperatura foram as que mais sofreram alterações em suas viscosidades. Piana et al. (2013), Coelho et al. (2016), Fialho et al. (2017), Deuschle et al. (2015) e Deuschle et al. (2018) também observaram maiores variações de viscosidade em suas formulações mantidas em temperaturas elevadas.

A viscosidade é diretamente afetada pela temperatura, e isso tem a ver com a proximidade das moléculas. Esse parâmetro é ligado à força de atração entre moléculas, ou seja, se a formulação se encontra em alta temperatura, as moléculas estão mais afastadas e isso consequentemente irá diminuir a viscosidade. Por outro lado, quando a temperatura é baixa, as moléculas tendem a ter mais atração, aumentando a viscosidade (ISAAC et al., 2008; CANCIAM, 2012; MELO et al., 2013).

Em geral, a formulação contendo apenas vitamina $E$ obteve os melhores resultados, pois não demonstrou alteração quanto às características sensoriais $\mathrm{e}$ ao fim dos 90 dias com uma viscosidade adequada. Esses aspectos demonstram estabilidade do produto $\mathrm{e}$ consequentemente a sua qualidade. Devido às características descritas na literatura em relação aos efeitos das substâncias ativas na pele, as formulações revelam potencial antioxidante, o que justifica seu emprego como cosméticos antienvelhecimento.

\section{CONCLUSÕES}

No presente estudo observou-se que a amostra que continha apenas vitamina $\mathrm{E}$ permaneceu estável a todos os ensaios de estabilidade realizados no período de 90 dias. Já as amostras contendo óleo de romã, seja como único ativo ou associado à vitamina E, apresentaram alterações de cor e odor a partir do trigésimo dia de armazenamento quando submetidas a alta temperatura. Todas as amostras mantidas em refrigerador e temperatura ambiente mantiveram-se estáveis quanto às características sensoriais e pH. Também, as amostras conservadas em temperatura ambiente não apresentaram redução de viscosidade durante o período de estudo. Desta forma, para garantir a estabilidade e, consequentemente, a qualidade das formulações, estas deverão ser conservadas em refrigerador ou até $25 \circ \mathrm{C}$ para manter as características originais do produto. $\mathrm{O}$ estudo, no entanto, encoraja futuras pesquisas em relação aos efeitos benéficos dessas formulações por meio de ensaios quantitativos para avaliação da manutenção do princípio ativo e efeito antioxidante, além de ensaios in vitro e in vivo.

\section{REFERÊNCIAS}

ALLEMANN, I. B.; BAUMANN, L. S. Antioxidantes e as formulações para cuidados com a pele. Revista RBM Especiais, v. 18, p. 18-14, 2012.

ALMEIDA, M. M. et al. Stability evaluation of tocopheryl acetate and ascorbyl tetraisopalmitate in isolation and incorporated in cosmetic formulations using thermal analysi. Brazilian Journal of Pharmaceutical Sciences, v. 46, n. 1, p. 129-134, 2010.

BABY, A. R. et al. Estabilidade e estudo de penetração cutânea in vitro da rutina veiculada em uma emulsão cosmética através de um modelo de biomembrana alternativa. Brazilian Journal of Pharmaceutical Sciences, v. 44, n. 2, p. 233248, 2008.

BARBA, J.; RIBEIRO, E. R. Efeito da microdermoabrasão no envelhecimento facial. Revista Inspirar, v. 1, n. 1, p. 6-9, 2009.

BARREIROS, A. L. B. S.; DAVID, J. M.; DAVID, J. P. Estresse oxidativo: relação entre geração de espécies reativas e defesa do organismo. Química Nova, v. 29, n. 1, p. 113-123, 2006.

BATISTELLA, M. A.; CHORILLI, M.; LEONARDI, G. R. Abordagens no estudo do envelhecimento cutâneo em diferentes etnias. Revista Brasileira de Farmácia, v. 88, n. 2, p. 59-62, 2007.

BRASIL. Agência Nacional de Vigilância Sanitária. Guia de Estabilidade de Produtos Cosméticos. Brasília, 2004.

BRASIL. Agência Nacional de Vigilância. Guia de Controle de Qualidade de Produtos Cosméticos. Brasília, 2008.

BUGNOTTO, C. et al. Estudo de estabilidade de formulação tópica contendo própolis. Disciplinarum Scientia. Série: Ciência e Saúde, v. 7, n. 1, p. 1-12, 2006.

CANCIAM, C. A. Efeito da temperatura na viscosidade de alguns ésteres: predição da energia de ativação viscosa. E-xacta, v. 5, n. 1, p. 83-97, 2012.

CHORILLI, M. et al. Aspectos gerais em sistemas transdérmicos de liberação de fármacos. Revista Brasileira de Farmácia, v. 88, n. 1, p. 7-13, 2007a.

CHORILLI, M. et al. Influência da viscosidade do veículo na liberação in vitro da cafeína. Revista Eletrônica de Farmácia, v. IV, n. 1, p. 52-60, 2007b.

COELHO, K. D. et al. Desenvolvimento e avaliação da estabilidade e capacidade antioxidante de uma formulação em gel contendo extrato das folhas de goiabeira (Psidium guajava L.). Biomotriz, v. 10, n. 1, p. 136-155, 2016.

DA ROSA, C. S. et al. Atividade antioxidante do ácido hialurônico extraído da crista de frango. Ciência Rural, v. 38, n. 9, p. 2593-2698, 2008. 
DEUSCHLE, V. C. K. N. et al. Physical chemistry evaluation of stability, spreadability, in vitro antioxidant, and photo-protective capacities of topical formulations containing Calendula officinalis L. leaf extract. Brazilian Journal of Pharmaceutical Sciences, v. 51, n. 1, p. 63-75, 2015.

DEUSCHLE, V. C. K. N. et al. Persea americana Mill. crude extract exhibits antinociceptive effect on UVB radiationinduced skin injury in mice. Inflammopharmacology, v. 2.018, p. 1-16, 2018.

FADAVI, A.; BARZEGAR, M.; AZIZI, H. M. Determination of fatty acids and total lipid content in oilseed of 25 pomegranates varieties grown in Iran. Food Science and Technology International, v. 11, n. 2, p. 113-119, 2005.

FARIA, A. B. et al. Desenvolvimento e avaliação de produtos cosméticos para a higiene capilar contendo tensoativos" não sulfatados". Revista de Ciências Farmacêuticas Básica e Aplicada, v. 33, n. 4, p. 521-527, 2012.

FIALHO, M. F. P. et al. Buddleja thyrsoides Lam. crude extract presents antinociceptive effect on an arthritic pain model in mice. Biochem J., v. 474, n. 17, p. 2.993-3.010, 2017.

FIGUEIREDO, B. K.; MARTINI, P. C.; MICHELIN, D. C. Desenvolvimento e estabilidade preliminar de um fitocosmético contendo extrato de chá verde (Camellia sinensis) (L.) Kuntze (Theaceae). Revista Brasileira de Farmácia, v. 95, n. 2, p. 770-788, 2014.

GUARATINI, T.; GIANETI, M. D.; CAMPOS, P. M. B. G. M. Stability of cosmetic formulations containing esters of Vitamins $\mathrm{E}$ and $\mathrm{A}$ : Chemical and physical aspects. International Journal of Pharmaceutics, v. 327, p. 12-16, 2006.

GUARATINI, T; MEDEIROS, M. H. G.; COLEPICOLO, P. Antioxidantes na manutenção do equilíbrio redox cutâneo: uso e avaliação de sua eficácia. Química Nova, v. 30, n. 1, p. 206213, 2007.

HARRIS, M. I. N. DE C. Pele-estrutura, propriedades e enveIhecimento. São Paulo: Senac, 2012.

HORA, J. J. et al. Chemopreventive effects of pomegranate seed oil on skin tumor development in CD1 mice. Journal of Medicinal Food, v. 6, n. 3, p. 157-61, 2003.

ISAAC, V. L. B. et al. Protocolo para ensaios físico-químicos de estabilidade de fitocosméticos. Revista de Ciências Farmacêuticas Básica e Aplicada, v. 29, n. 1, p. 81-96, 2008.

KAMMEYER, A.; LUTIEN, R. M. Oxidation events and skin aging. Ageing Research Reviews, v. 21, p. 16-29, 2015.

LANGE, K. M.; HEBERLÉ, G.; MILÃO, D. Avaliação de estabilidade e atividade antioxidante de uma emulsão base não-iônica contendo resveratrol. Brazilian Journal of Pharmaceutical Sciences, v. 45, n. 1, p. 145-151, 2009.

LEONARDI, G. R.; GASPAR, L. R.; CAMPOS, P. M. B. G. M. Estudo da variação do $\mathrm{pH}$ da pele humana exposta à formulação cosmética acrescida ou não das vitaminas $A$, $E$ ou de ceramida, por metodologia não invasiva. Anais Brasileiros de Dermatologia, v. 77, n. 5, p. 563-569, 2002.

LYRIO, E. S. et al. Recursos vegetais em biocosméticos: conceito inovador de beleza, saúde e sustentabilidade. Natureza on-line, v. 9, n. 1, p. 47-51, 2011.
MELO, E. K. S. et al. Análise e estudo viscosimétrico de diferentes géis de cetoprofeno 2,5\%. Revista de Ciências Farmacêuticas Básica e Aplicada, v. 34, n. 1, p. 95-99, 2013.

MOTTA, M. P.; FIGUEIREDO, P. A.; DUARTE, J. A. Teorias biológicas do envelhecimento. Revista Portuguesa de Ciências do Desporto, v. 4, n. 1, p. 81-110, 2004.

MOUAD, A. M.; PORTO, A. L. M. Uma abordagem química sobre a pele e a biocatálise no desenvolvimento de moléculas antioxidantes de aplicação cosmética. Revista Virtual de Química, v. 6, n. 6, p. 1.642-1.660, 2014.

ORESAJO, C. et al. Antioxidants and the skin: understanding formulation and efficacy. Dermatology and Therapy, v. 25, n. 3, p. 252-259, 2012.

OZGUL-YUCEL S. Determination of conjugated linolenic acid content of selected oil seeds grown in Turkey. Journal of the American Oil Chemists' Society, v. 82 n. 12, p. 893-897, 2005.

PIANA, M. et al. Antiinflammatory effects of Viola tricolor gel in a model of sunburn in rats and the gel stability study. Journal of Ethnopharmacology, v. 150, n. 2, p. 458-465, 2013.

SANTOS, M. P.; OLIVEIRA, N. R. F. Ação das vitaminas antioxidantes na prevenção do envelhecimento cutâneo. Disciplinarum Scientia, Série: Ciências da Saúde, v. 15, n. 1, p. 75-89, 2013.

SCHNEIDER, C. D.; OLIVEIRA, A. R. Radicais livres de oxigênio e exercício: mecanismos de formação e adaptação ao treinamento físico. Revista Brasileira de Medicina do Esporte, v. 10, n. 4, p. 308-313, 2004.

SILVA JUNIOR, E. et al. Formação de nanoemulsões do tipo óleo em água contendo óleo de semente de romã. Disciplinarum Scientia-Naturais e Tecnológicas, v. 14, n. 1, p. 115122, 2013.

SINIGAGLIA, G. et al. Iontoforese associada ao princípio ativo ácido ascórbico: Avaliação de difusão vertical "in vitro". Scientia Plena, v. 10, n. 4, p. 1-8, 2014.

SOARES, S. E. Ácidos fenólicos como antioxidantes. Revista de Nutrição, v. 15, n. 1, p. 71-81, 2002.

STRUTZEL, E. et al. Análise dos fatores de risco para o enveIhecimento da pele: aspectos gerais e nutricionais. Revista Brasileira de Nutrição Clínica, v. 22, n. 2, p. 139-145, 2007.

TESTON, A. P.; NARDINO, D.; PIVATO, L. Envelhecimento cutâneo: teoria dos radicais livres e tratamentos visando a prevenção e o rejuvenescimento. Uningá Review, v. 1, n. 1, p. 71-84, 2010.

VIEIRA, R. P. et al. Physical and physicochemical stability evaluation of cosmetic formulations containing soybean extract fermented by Bifidobacterium animalis. Brazilian Journal of Pharmaceutical Science, v. 45, n. 3, p. 515-525, 2009. VIUDA-MARTOS, M.; FERNÁNDEZ-LÓPEZ, J.; PÉREZ-ÁLVAREZ, J. A. Pomegranate and its Many Functional Components as Related to Human Health: A Review. Comprehensive Reviews in Food Science and Food Safety, v. 9, n. 6, p. 635-654, 2010. 